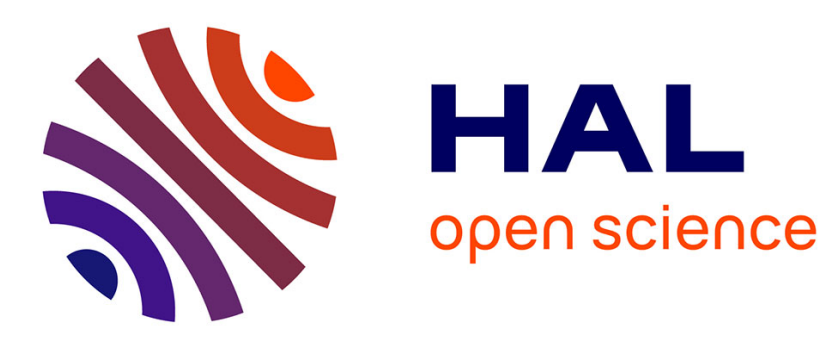

\title{
Internal Model Control with Distributed-Delay-Compensator to Attenuate Multi-Harmonic Periodic Disturbance of Time-Delay System
}

Can Kutlu Yuksel, Jaroslav Busek, Tomas Vyhlidal, Silviu-Iulian Niculescu, Martin Hromcik

\section{To cite this version:}

Can Kutlu Yuksel, Jaroslav Busek, Tomas Vyhlidal, Silviu-Iulian Niculescu, Martin Hromcik. Internal Model Control with Distributed-Delay-Compensator to Attenuate Multi-Harmonic Periodic Disturbance of Time-Delay System. IEEE 60th Conference on Decision and Control (CDC 2021), IEEE, Dec 2021, Austin, TX, United States. hal-03457093

\section{HAL Id: hal-03457093 \\ https://hal-centralesupelec.archives-ouvertes.fr/hal-03457093}

Submitted on 30 Nov 2021

HAL is a multi-disciplinary open access archive for the deposit and dissemination of scientific research documents, whether they are published or not. The documents may come from teaching and research institutions in France or abroad, or from public or private research centers.
L'archive ouverte pluridisciplinaire HAL, est destinée au dépôt et à la diffusion de documents scientifiques de niveau recherche, publiés ou non, émanant des établissements d'enseignement et de recherche français ou étrangers, des laboratoires publics ou privés. 


\title{
Internal Model Control with Distributed-Delay-Compensator to Attenuate Multi-Harmonic Periodic Disturbance of Time-Delay System*
}

\author{
Can Kutlu Yuksel, Jaroslav Busek, Tomas Vyhlidal\#, Silviu-Iulian Niculescu, Martin Hromcik
}

\begin{abstract}
A distributed-delay based compensator for the Internal Model Control scheme is introduced as an alternative to the repetitive control structure to attenuate periodic disturbance with multiple harmonics. Besides the periodic signal attenuation, the scheme is derived to compensate an input time-delay of the controlled system. Next, the performance requirements on the spectral, frequency, and time domain characteristics are used for an optimization-based design. The task is to minimize the $\mathcal{H}_{\infty}$ norm of the weighted sensitivity, while the spectral and structural properties comply with the constraints. The proposed design is tested on a model of a hot rolling process, which motivates the research. Numerical analysis and simulation-based verification complete the presentation.
\end{abstract}

\section{INTRODUCTION}

Many industrial processes are in some way required to work under periodic actions either due to carrying out a repetitive task or due to a periodic disturbance caused by a rotating or vibrating mass present in the working environment. In both cases, such processes require controllers to achieve and sustain their goals. Arguably the most popular technique to design controllers that work with periodic signals is the repetitive control strategy. This control strategy has been widely studied and proved its effectiveness in various fields. In this paper, we propose an alternative controller design method, motivated by the repetitive control paradigms, that allows a wide range of systems to work under periodic actions. The design is based on the Internal Model Control (IMC) and makes use of a distributed delay for the compensation of periodic signals. Additionally, the control system is designed to simultaneously address the substantial input delay present in the plants. Such a design consideration stems from the goal to remove the periodic surface defects

*This work was supported by the Czech Science Foundation project No. 21-07321S - Persistent problems of repetitive control. The work of the first and fourth author was also supported by a public grant overseen by the French National research Agency (ANR) as part of the « Investissements d'Avenir » program, through the "ADI 2020" project funded by the IDEX Paris-Saclay, ANR-11-IDEX-0003-02. The first author was also supported by the Grant Agency of the Czech Technical University in Prague, grant No. SGS20/159/OHK2/3T/12.

T. Vyhlidal (\# corresponding author), C.K. Yuksel and J. Busek are with Dept. of Instrumentation and Control Eng., Faculty of Mechanical Engineering, Czech Technical University in Prague, Technická 1902/4, 16607 Praha 6, Czech Republic, Tomas.Vyhlidalefs.cvut.cz

C.K. Yuksel and S-I Niculescu are with Université Paris-Saclay, CNRS, CentraleSupélec, Inria, Laboratoire des Signaux et Systèmes, 91192 Gif-sur-Yvette, France Silviu.Niculescuel2s. centralesupelec.fr

Martin Hromcik is with the Department of Control Engineering, Faculty of Electrical Engineering, Czech Technical University in Prague, Karlovo namesti 1312135 Praha 2, Czech Republic Martin.Hromcik@fel.cvut.cz observed in hot rolling mills which suffer from input timedelays.

\section{A. Repetitive Control - motivation and solutions}

Repetitive control was initially introduced by Inoue [1] and it is based on the Internal Model Principle stated by Francis and Wonham [2]. For periodic exogenous signals, the controller design paradigm discussed in [2] is generalized by employing the periodic signal generator

$$
D_{\mathrm{PSG}}(s)=\frac{1}{1-e^{-s T_{d}}}
$$

which generally represents all the periodic signals with a certain period $T_{d}$. In this setting, the controller is referred to as the repetitive controller. However, with the time-delays occurring in the closed-loop, such a feature for applications under periodic actions made the design of stabilizing controllers rather difficult. The initial design introduced by Inoue was proven to be applicable to only SISO proper systems with BIBO stability and its application to strictly proper systems was made possible by the modified repetitive controller introduced in [3]. Such a controller consists of a low-pass filter associated with the delay term in the periodic signal generator as shown in Fig. 1, and its sensitivity transfer

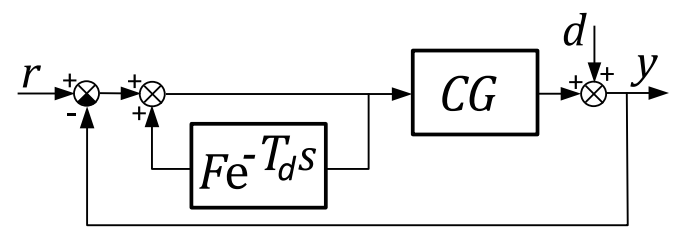

Fig. 1. Repetitive Control Scheme

function is expressed by

$$
S(s)=\frac{1-F(s) e^{-s T_{d}}}{1-F(s) e^{-s T_{d}}+C(s) G(s)} .
$$

In the ideal case when $F(s)=1$, the transfer function has infinitely many zeros satisfying $1-e^{-s T_{d}}=0$. In such a case, the zeros lie on the imaginary axis at the integer multiples of the frequency of the periodic signal generator i.e $s_{k}=j \frac{2 \pi k}{T_{d}}, k \in \mathbb{Z}$. Since a periodic signal with a period $T_{d}$ can be expressed by its Fourier series as

$$
d(t)=\frac{c_{0}}{2}+\sum_{l=1}^{M} c_{l} \cos \left(\frac{2 \pi l}{T_{d}} t-\varphi_{l}\right),
$$

with $c_{l}, \varphi_{l}, l=1, \ldots, M$ determining the amplitudes and phase shifts of the $M$ harmonic components and $M$ being, 
in general, infinite, it follows that the poles of the periodic signal are cancelled by the zeros of the sensitivity transfer function. This fact leads the error to converge to zero provided that the closed-loop is stable. However, in this setting, the dynamics of the closed-loop system are described by neutral functional differential equations (see, e.g. [4], [5] and the references therein), having both point and essential spectrum in the linear case that makes the analysis more complicated and further involved. The introduction of the low-pass filter $F(s)$ addresses this issue. Even though the filter causes the harmonic zeros to shift away from the imaginary axis at high-frequencies and, therefore, limits the precision of the signal generation, this downgrade is justified by the fact that the overall closed-loop is represented now by a set of retarded functional differential equations possessing only point spectrum in the linear case. In other words, this last setting requires less strict conditions for stabilization.

Additionally, in terms of practical applications, this loss of precision does not influence the applicability of the control scheme since the level of precision can be tuned by the passband of the filter.

After assessing the stability for the initial case, the development of repetitive control continued at three main directions: (i) enhancing reference tracking or disturbance rejection performance of periodic and non-periodic signals, (ii) improving the robustness of the whole control system against frequency uncertainties, noises and identification errors, and, (iii) extending the application of repetitive control to advanced systems like non-linear, time-delayed and MIMO systems and systems with control constraints.

Despite the benefit it introduces for tracking and rejecting periodic signals, the inclusion of the periodic signal generator into closed-loop also negatively affects the tracking and rejection of non-periodic signals. To address this issue, Inoue in [6] introduced the high-order repetitive controller in which the time-delay component $e^{-T_{d} s}$ is replaced by the polynomial $W(s)=\sum_{i=1}^{m} w_{i} e^{-m s T_{d}}$ where $w_{i}$ denotes the weight of the $i^{\text {th }}$ term. It was shown that the tracking performance for aperiodic signals can be improved by tuning the weights. A method for tuning the weights using $\mathcal{H}_{\infty}$ norm was proposed in [7] which resulted in a better performance than what is proposed in [6]. The degree of freedom introduced by the weights led to many advances for the robustness of repetitive control as well.

The robustness of the repetitive control is mainly investigated for two sources of uncertainties. One of them is the uncertainty arisen from the inaccuracy between the plant and the model. The second one is the uncertainty imposed by the varying or unknown period of an exogenous signal. For repetitive control systems suffering from model errors, their performance and robustness were studied using structured singular values in [8]. A graphical design technique that ensured the stability of the control system despite plant uncertainties was proposed in [9] and repetitive control for linear systems with time-varying and norm-bounded uncertainties was studied in [10] using Lyapunov functionals for time-delay systems. For systems with input-delay, a robust repetitive controller was designed in [11] based on $\mu$ synthesis.

On the other hand, robustness against variations and uncertainties in the period of the exogenous signal holds great importance for the reliability of the repetitive control. The effectiveness of the repetitive control relies on matching the zeros on the imaginary axis introduced by the periodic signal generator with the poles of the exogenous periodic signal. Therefore, the controller should be able to tolerate some uncertainty in the period of the signal to prevent failures. In [12], [13] an adaptive repetitive control based on the discretetime setting was proposed for tracking periodic signals with an unknown period. A genetic algorithm-based repetitive control design was studied in [14]. Steinbuch initially studied the high-order repetitive controller to improve the robustness of the repetitive control to signals with uncertain period in [15] and later on in [16] described a unifying method that covered the results from [7] and [16]. An LMI based design method that takes into account both the robustness for period uncertainty and non-periodic signal performance was proposed in [17].

\section{B. Distributed Delays in Control and Compensation}

As it will be shown, involving an IMC scheme in the periodic signal compensation provides a straightforward controller design and enables the input delay compensation. Nevertheless, the main contribution of this paper lies in involving a distributed delay instead of the standard lumped delay in the harmonics compensation. This research direction builds up on the results of the broader authors team gained from the application of distributed delay in input shaping [18], [19], [20], [21] and vibration control, [22], [23], [24]. The main benefit of involving the distributed delay stems from the retarded spectral features at the high-frequency range. Compared to the neutral dynamics brought by the lumped delay, no further filtering is needed to save the stability at high frequencies.

The paper is organised as follows. In Section II, the IMC scheme with the main components is proposed and adjusted towards compensating both the input time delay and the periodic disturbance. The compensator design based on the distributed delay, which is the main proposition of the paper, is presented in Section III. Section IV presents a practically motivated case study example. Brief conclusions and further directions are highlighted in Section V.

\section{Internal Model Control ANd COMPENSATION SCHEME}

In order to fulfill the two compensation tasks (periodic disturbance and input time delay), an Internal Model Control (IMC) scheme is considered as shown in Fig. 2. The IMC arrangement was first introduced and carefully studied by Garcia and Morari [25]. Its design and performance under periodic disturbances were studied in [26] and its application to first order systems with time-delay was studied by Vyhlidal and Zitek in [27], see also [28]. 
The controlled plant in the IMC scheme in Fig. 2 is considered to be in the form

$$
G(s)=\frac{y(s)}{u(s)}=G_{i}(s) \mathrm{e}^{-s \tau}
$$

where $u, y$ are the system input and output, respectively; $G_{i}(s)$ is assumed to be a proper and invertible transfer function, i.e. without any positive zeros, and $\tau$ is the input delay to be compensated. The transfer function $G_{m}(s) \mathrm{e}^{-s \tau_{m}}$ denotes the model of the plant with input delay $\tau_{m}$. In the nominal form, it is assumed that $G_{m}(s)=G_{i}(s)$ and $\tau_{m}=\tau$.

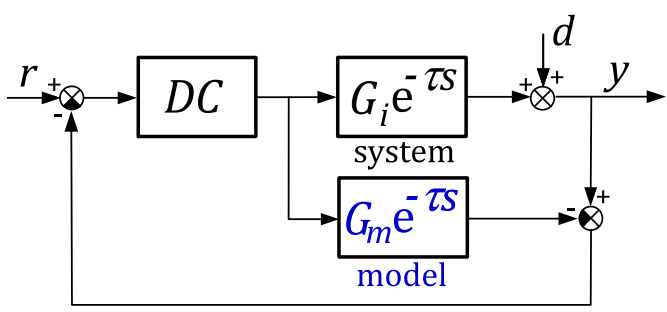

Fig. 2. Proposed internal model control scheme for periodic disturbance compensation

As it will be shown, the delay $\tau$ compensation is imposed by the IMC scheme. The compensation of the multiharmonic periodic disturbance $d(s)$ is performed by the compensator $D$, which is of a distributed-delay form with an overall length $T_{D}$. The compensator output $\bar{u}$ is determined by

$$
\bar{u}(t)=\int_{0}^{T_{D}} D(\eta) e(t-\eta) \mathrm{d} \eta
$$

where $e$ is the compensator input. Analogous to the input shaper application [20], the delay is assumed to be piece-wise equally distributed and expressed by the transfer function

$$
D(s)=\frac{1}{s} \sum_{k=0}^{N} a_{k} e^{-s k \vartheta}
$$

where $\vartheta=\frac{T_{D}}{N}$ and $N+1$ is the number of coefficients $a_{k}$ to be tuned.

The last component of the IMC scheme in Fig. 2 is the controller given by

$$
C(s)=\frac{1}{G_{m}(s)} F(s),
$$

where $F(s)$ is a low-pass filter included to guarantee that $D(s) C(s)$ is a proper (biproper, at least) transfer function satisfying

$$
\lim _{s \rightarrow 0} F(s)=1,
$$

i.e. having unity static gain.

The sensitivity function of the scheme in Fig. 2 is given by

$$
\begin{aligned}
S(s)= & \frac{y(s)}{d(s)}= \\
& \frac{1-D(s) C(s) G_{m}(s) \mathrm{e}^{-s \tau_{m}}}{1+D(s) C(s)\left(G_{i}(s) \mathrm{e}^{-s \tau}-G_{m}(s) \mathrm{e}^{-s \tau_{m}}\right)} .
\end{aligned}
$$

With the assumption $G_{i}(s)=G_{m}(s)$ and the controller in the form (7), it reduces to

$$
S(s)=1-D(s) F(s) \mathrm{e}^{-s \tau_{m}},
$$

which will be further considered for the design. Note that for this nominal case, the complementary sensitivity function is given by

$$
T(s)=D(s) F(s) \mathrm{e}^{-s \tau_{m}} .
$$

Notice that in the nominal form (10), the input delay $\tau$ has been fully compensated and the dynamics is finite dimensional. However, this is a strong idealization and therefore, in practice, the closed loop dynamics still needs to be considered as infinite dimensional with the characteristic equation given by

$$
1+C(s) D(s)\left(G_{i}(s) \mathrm{e}^{-s \tau}-G_{m}(s) \mathrm{e}^{-s \tau_{m}}\right)=0
$$

which can be further simplified to

$$
G_{m}(s)\left(1-D(s) F(s) \mathrm{e}^{-s \tau_{m}}\right)+D(s) F(s) G_{i}(s) \mathrm{e}^{-s \tau}=0 .
$$

Taking into account that all the involved transfer functions are proper, thanks to the distributed delay features recognised in [20], the closed loop dynamics is of retarded time delay form with all the positive spectrum distribution features [4], [20].

\section{Distributed-DELAY BASED COMPENSATOR DESIGN}

The distributed delay $D(s)$ is to be shaped with the objective to compensate the dominant (low-frequency) harmonics of the periodic disturbance $d(t)$. Taking into account the Fourier series expansion (3), the compensation of $M_{d}$ dominant harmonics with frequencies $\Omega_{l}=\frac{2 \pi l}{T_{d}}, l=1,2, . ., M_{d}$ leads to the equation set

$$
S\left(j \Omega_{l}\right)=0, l=1,2, . . M_{d} .
$$

Reflecting this to the nominal sensitivity function (10) substituted with the compensator in (6), we get

$$
\sum_{k=0}^{N} a_{k} e^{-j \Omega_{l} k \vartheta}=\frac{j \Omega_{l}}{F\left(j \Omega_{l}\right)} \mathrm{e}^{j \Omega_{l} \tau_{m}}, l=1,2, . . M_{d} .
$$

When each equation is split to its real and imaginary part, we obtain

$$
\begin{aligned}
& \sum_{k=0}^{N} a_{k} \cos \left(\Omega_{l} k \vartheta\right)=R_{l}, \\
& \sum_{k=0}^{N} a_{k} \sin \left(\Omega_{l} k \vartheta\right)=I_{l},
\end{aligned}
$$

where

$$
R_{l}=\Re\left(\frac{j \Omega_{l} e^{j \Omega_{l} \tau_{m}}}{F\left(j \Omega_{l}\right)}\right), I_{l}=-\Im\left(\frac{j \Omega_{l} e^{j \Omega_{l} \tau_{m}}}{F\left(j \Omega_{l}\right)}\right)
$$

for $l=1,2, \ldots, M_{d}$.

Additional conditions to assess the parameters $a_{i}$ result from the performance and structural requirements on the compensator. Taking into account the requirements on the 
reference tracking $\left(\lim _{s \rightarrow 0} T(s)=1\right)$ and the disturbance rejection $\left(\lim _{s \rightarrow 0} S(s)=0\right.$ ) and considering (8), the compensator needs to have a static unity gain, i.e.

$$
\lim _{s \rightarrow 0} D(s)=1 \text {. }
$$

For the structure in (6), this leads to the condition

$$
\sum_{k=0}^{N} a_{k}=0
$$

implying the finite response feature of $D(s)$ and additionally to the condition

$$
\sum_{k=0}^{N} a_{k} k \vartheta=-1
$$

Thus, taking into account the equation set (16), (19) and (20) the design problem of $D(s)$ can be formulated as a solution of

$$
A x=B,
$$

where $x \in \mathbb{R}^{N+1}, A \in \mathbb{R}^{2 M_{d}+2 \times N+1}, B \in \mathbb{R}^{2 M_{d}+2}$, given as $x=\left[a_{0}, a_{1}, \ldots, a_{N}\right]^{\mathrm{T}}$,

$$
A=\left[\begin{array}{cccc}
1 & \cos \left(\Omega_{1} \vartheta\right) & \cdots & \cos \left(\Omega_{1} N \vartheta\right) \\
\vdots & \vdots & \cdots & \vdots \\
1 & \cos \left(\Omega_{M_{d}} \vartheta\right) & \cdots & \cos \left(\Omega_{M_{d}} N \vartheta\right) \\
0 & \sin \left(\Omega_{1} \vartheta\right) & \cdots & \sin \left(\Omega_{1} N \vartheta\right) \\
\vdots & \vdots & \cdots & \vdots \\
0 & \sin \left(\Omega_{M_{d}} \vartheta\right) & \cdots & \sin \left(\Omega_{M_{d}} N \vartheta\right) \\
1 & 1 & \cdots & 1 \\
0 & \vartheta & \cdots & N \vartheta
\end{array}\right], B=\left[\begin{array}{c}
R_{1} \\
\vdots \\
R_{M d} \\
I_{1} \\
\vdots \\
I_{M d} \\
0 \\
-1
\end{array}\right]
$$

Assuming $N+1>M_{d}$, equation (21) has infinitely many solutions and can be solved via the pseudoinverse

$$
x=\left(A^{\mathrm{T}} A\right)^{-1} A^{\mathrm{T}} B,
$$

yielding the least squares solution of (21). It should be stressed that the solution of (21) requires $A$ being of full row rank. This is fulfilled for any parameters satisfying

$$
2 \frac{M_{d}}{T_{d}}<\frac{N}{T_{D}}
$$

Under this condition the arguments of sine and cosine functions in the matrix $A$ given by $\Omega_{l} k \vartheta=\frac{2 \pi l}{T_{d}} \frac{k T_{D}}{N}, k=1 . . N$ are sufficiently small to avoid any dependency between the rows of $A$.

In what follows, considering that $N+1>>M_{d}$, we introduce additional positive features for the design of the compensator.

\section{A. Optimal design}

In order to enhance the closed loop robustness, we employ the standard $\mathcal{H}_{\infty}$-norm minimization of the weighted sensitivity function [29]. Considering the above derived conditions (21), the design is formulated as a constrained optimization problem

$$
\begin{array}{cc}
\min _{x} & \left\|\left(1-D(j \omega) F(j \omega) e^{-j \omega \tau_{m}}\right) W(j \omega)\right\|_{\infty} \\
\text { s.t. } & A x=B \\
& A_{n} x \leq B_{n}
\end{array}
$$

where

$$
W(s)=\frac{\frac{1}{b_{u}} s+\omega_{b}}{s+b_{l} \omega_{b}}
$$

is the weight function determining the desired bandwidth $\omega_{b}$, lower $b_{l}$ and upper $b_{u}$ bounds of the sensitivity function.

The involved inequality constraint in (24) is used to impose the limits on the Jerk, i.e. maximum $J_{\max }$ and minimum $J_{\min }$ of the impulse response of $D(s)$, which results to

$$
A_{n}=\left[\begin{array}{c}
L \\
-L
\end{array}\right], B_{n}=\left[\begin{array}{c}
J_{\max } e_{N} \\
-J_{\min } e_{N}
\end{array}\right],
$$

where $L \in \mathbb{R}^{N+1 \times N+1}$ is a lower triangular matrix with $L_{p, q}=1$, for $q \leq p$ and $L_{p, q}=0$ otherwise, $e_{N} \in \mathbb{R}^{N+1}$ is the unity vector $e_{N}=[1,1, \ldots, 1]^{\mathrm{T}}$.

When solving the problem (24), one needs to take into account that it is performed over a frequency response of a time delay system for which even the computation of $\mathcal{H}_{\infty}$ norm is not straightforward [30] and built-in computational tools are not available, e.g. in Matlab. In general, the norm is a non-convex and non-smooth function with respect to the parameters [31]. Therefore, the problem (24) needs to be handled as a constrained nonlinear optimization problem. In the application example below, the Matlab fmincon is used with the initial condition resulting from (22). Alternatively, HANSO [32], (or recently designed GRANSO [33]), can be applied analogously to [31].

\section{Case Study Application to Hot Rolling PROCESS}

The above proposed control design is demonstrated on a hot rolling application, which motivates the research. The overall scheme of the hot rolling process is in Fig. 3. Structurally, it consists of working rolls that are in contact with the rolled plate and the back-up rolls being actuated by the hydraulic system. Since the rolled plate thickness is measured at a distance $L$ from the roll working point, the process inherits a time delay $\tau=\frac{L}{v}$, where $v$ is the plate velocity, considered constant here. One of many disturbances acting on the thickness is the periodic disturbance caused by the eccentricity of the rolls. To remove this effect, the hydraulic system responsible for moving the top roller in a vertical direction must be controlled accordingly.

In Fig. 4, an instance of measured process data is shown. As also shown, it can be well approximated by the first-order model with an input delay

$$
G(s)=G_{m}(s) e^{-\tau_{m} s}=\frac{h(s)}{H(s)}=\frac{K_{m}}{T_{m} s+1} e^{-\tau_{m} s}
$$

with $K_{m}=0.5, T_{m}=0.8 \mathrm{~s}, \tau_{m}=3.8 \mathrm{~s}$ describing the dynamics between the rolling gap $u(t)=H(t)$ and the measured plate thickness $y(t)=h(t)$. The frequency of the observed disturbance from the measured data is evaluated by FFT as $\Omega_{1}=5.3 \mathrm{~s}^{-1}$. Next to this lowest harmonic determining the period of the oscillations $T_{d}=1.1855 \mathrm{~s}$, there exists one more distinct harmonic observed from the measured data at $\Omega_{2}=10.6 \mathrm{~s}^{-1}$. In order to demonstrate the 


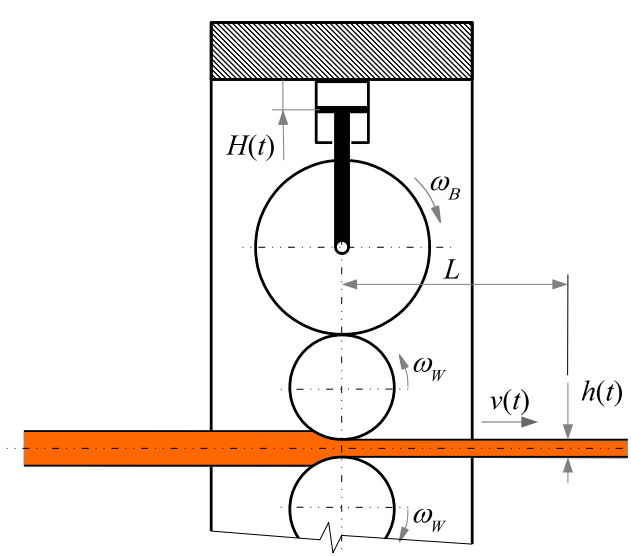

Fig. 3. Scheme of the rolling mill with working and back-up rolls.

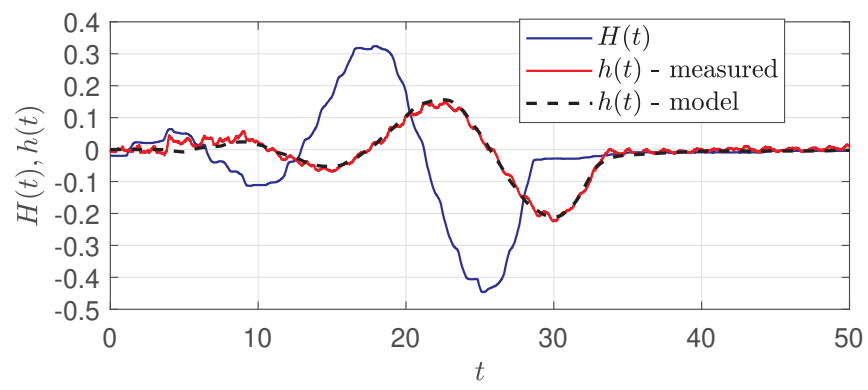

Fig. 4. Measured rolling process data and their fitting by the model (26)

potential of the proposed method, instead of targeting the relatively simple period formation from the real process, we target a more complex artificially generated period profile depicted in Fig. 5. In the bottom part of Fig. 5, the amplitudes of the harmonics are shown considering the signal decomposition by (3). In particular, eight amplitudes can be detected as dominant, which will be considered in the assigned $\Omega_{l}=l 5.3, l=1 . .8$, i.e. $M_{d}=8$.

Taking into account the length of the input delay and the oscillation period, the action length of the compensator is considered as $T_{D}=2 T_{d}=2.3710 \mathrm{~s}$. To assign the eight roots to $D(s)$ by (16) and satisfy the additional conditions (19) and (20), at least 18 parameters $a_{k}$ are required. In order to have a sufficient degree of freedom to solve (24), we select $N=60$. The parameters of the weight function (25) are selected as $\omega_{b}=0.12 \mathrm{~s}^{-1}, b_{u}=2, b_{l}=0.01$ in order to achieve standard stability margin and low-frequency pass band compared to $\Omega_{1}$. The definition of the optimization problem is finalized by selecting $J_{\min }=-3$ and $J_{\max }=8$. The asymmetric Jerk limits are used with the objective to limit the possible step response undershoot as much as possible. Last but not least, the filter $F(s)$ needs to be selected for the controller

$$
C(s)=\frac{T_{m} s+1}{K} F(s) .
$$

In order to demonstrate the structural benefits of $D(s)$ in the distributed delay form (6), the minimal controller order is selected with $F(s)=1$. Interestingly, this option is possible
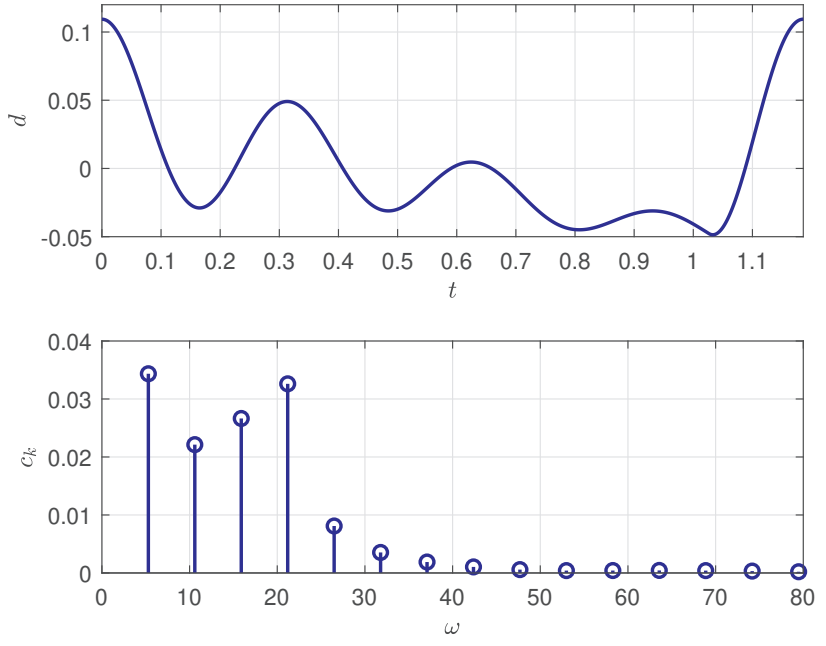

Fig. 5. Period of the disturbance $d$ and magnitudes of the harmonics according to (3).

when restructuring $D(s) C(s)$ to the form

$$
D(s) C(s)=\sum_{k=0}^{N} a_{k} e^{-s k \vartheta} \frac{T_{m} s+1}{K_{m} s},
$$

i.e. it is composed of a weighted sum of delayed inputs and a biproper transfer function. Thus, the non-delayed dynamics $G_{m}$ is completely compensated and the nominal dynamics is determined by the distributed delay only.
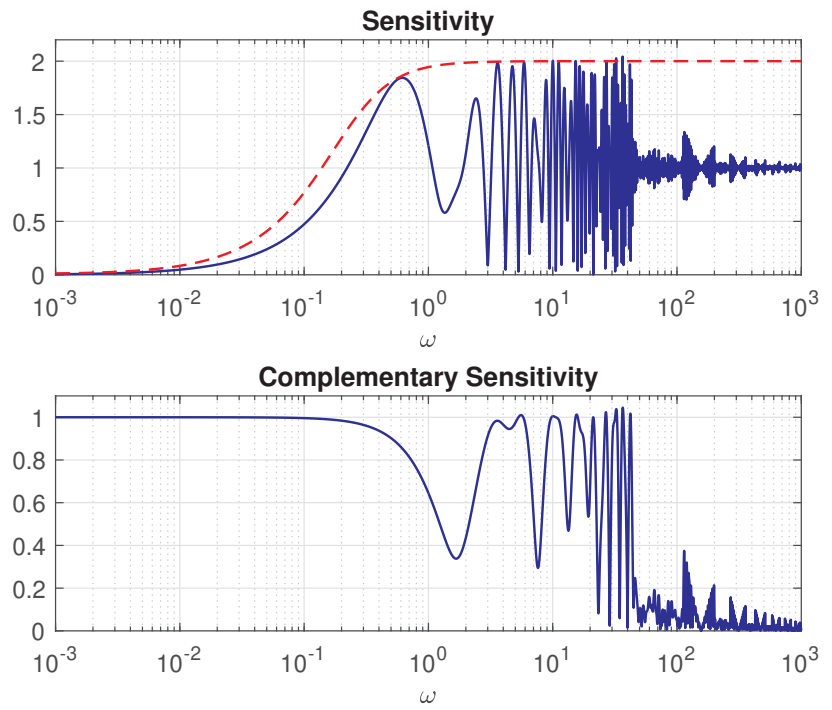

Fig. 6. Amplitude frequency responses of the sensitivity (10), complementary sensitivity (11) and the predefined weight $\frac{1}{W(s)}, W(s)$ given by (25)

The results of the optimization task, solved by fmincon are shown in Fig. 6. As can be seen, the requirements imposed on the nominal sensitivity function by (25) have been satisfied. Note that the $\mathcal{H}_{\infty}$ norm was determined numerically by sweeping the frequency over the frequency band visualised in Fig. 6. Despite of this, the overall evaluation time by fmincon was close to 20s. The result of the parameter 


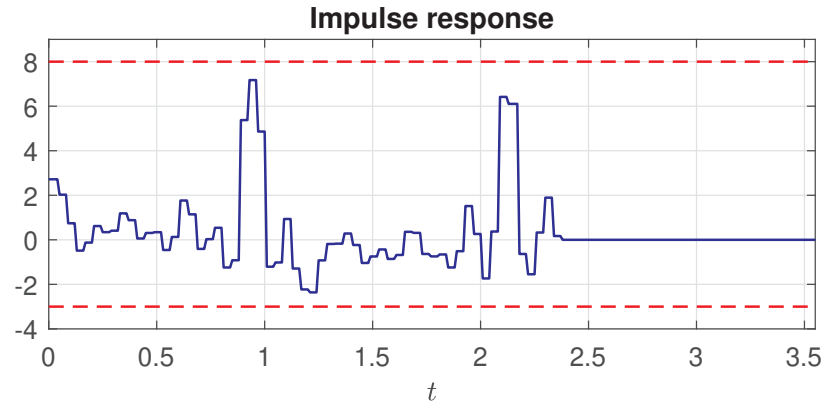

Fig. 7. Impulse Response of the distributed-delay-compensator $D(s)$ given by (6) with the imposed limits $J_{\min }$ and $J_{\max }$ (dashed).

optimization is presented visually in Fig. 7, demonstrating the predefined finite length and satisfied Jerk limits of the $D(s)$ impulse response. Simulation based verification of considered periodic disturbance compensation is shown in Fig. 8. It nicely demonstrates that after the transition time, not much longer than $\tau_{m}+T_{D}$, the dominant $M_{d}$ harmonics are fully compensated. The residual vibrations, lying below the expected noise level, corresponds to the higher frequency harmonics which have not been targeted.
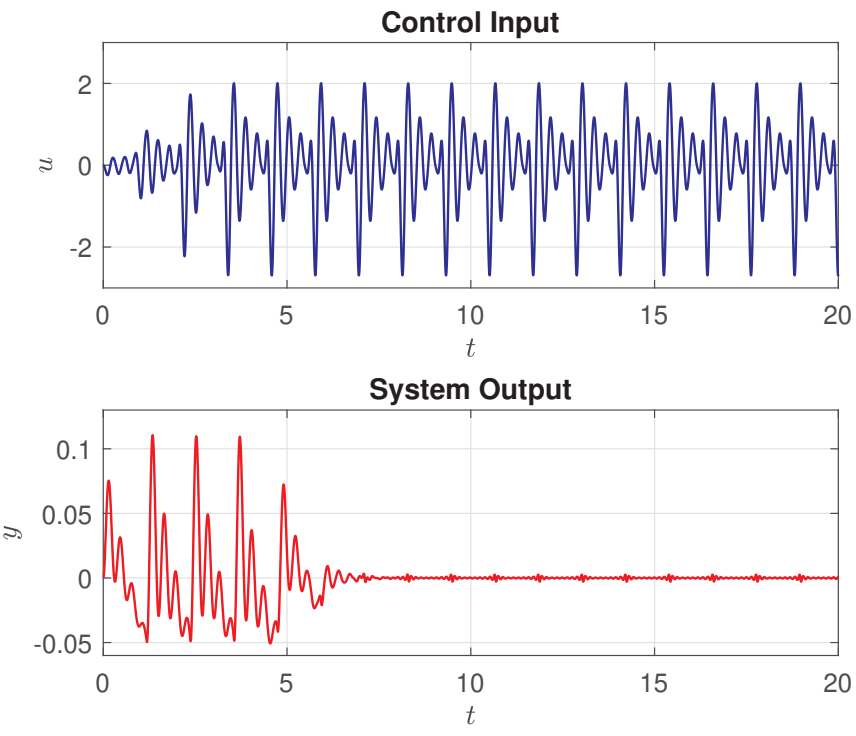

Fig. 8. Simulation based demonstration of the periodic disturbance compensation with the $d(s)$ period shape in Fig. (5), considering the nominal system $G_{i}(s)=G_{m}(s)$.

Next, the fulfillment of the equality constraints (16) is demonstrated in Fig. 9, where the spectrum of (10) zeros is shown, evaluated by QPmR [34]. As required, eight zeros have been placed at the predefined positions on the imaginary axis. Note that the zero in the origin is virtual as it is cancelled out by the $D(s)$ pole at the origin. Notice also that the retarded chain of infinitely many unassigned zeros is located on the left half complex plane. This property is crucial for the spectrum distribution of the infinitely many poles of the closed loop system which emerge under the model perturbation. Considering the perturbed plant model

$$
G_{i}(s)=\frac{0.9}{0.1 s+1} G_{m}(s),
$$

the system poles given as solutions of the equation (13) tend to match the spectrum of $S(s)$ zeros at the higher frequency range, as also shown in Fig. 9. Even though the perturbation affected the disturbance compensation, see Fig. 10, all the poles are located to the left and the controlled variable $y(t)$ asymptotically approaches to the reference $r=0$.
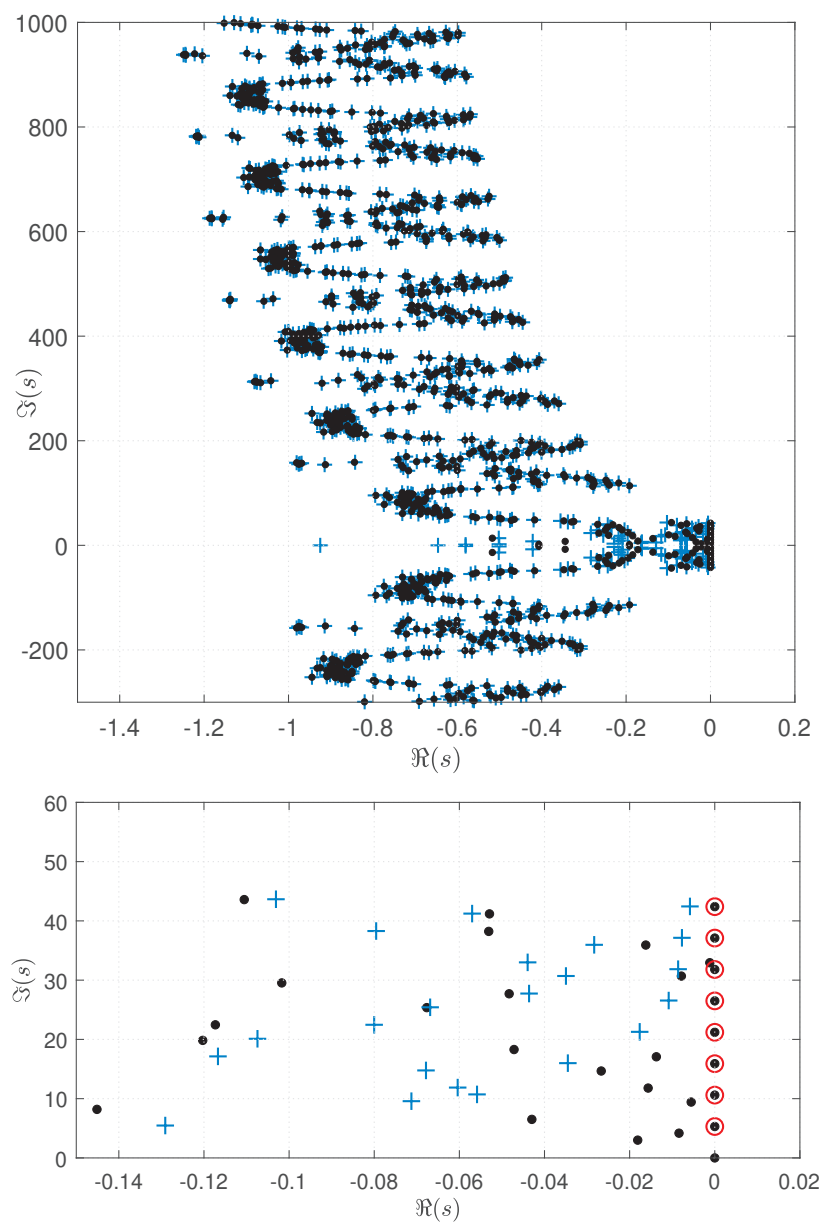

Fig. 9. Spectrum of zeros of the nominal sensitivity function (10) $(\bullet)$ and spectrum of poles of the perturbed closed loop system (+). (Top) Large scale distribution of spectra. (Bottom) Detailed distribution of spectra with the zeros placed at desired positions (red $\circ$ ).

\section{CONCLUSiON}

A complex design approach has been proposed to compensate the input time delay and the multi-harmonic periodic disturbance. Compared to the standard repetitive control, the proposed approach is straightforward in the sense that both the controller and the compensator design are performed simultaneously. As demonstrated in the case-study example, a large number of zeros corresponding to the active harmonics can be fully covered robustly even for a system with substantial input time delay and a certain level of plant parameter uncertainty. Most of the positive features arise 

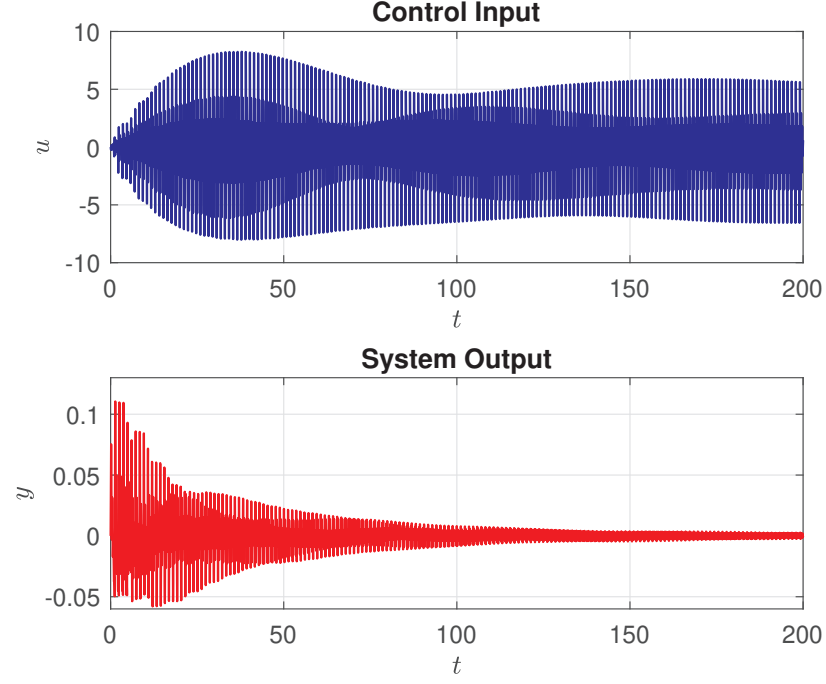

Fig. 10. Simulation based demonstration of the periodic disturbance compensation with the $d(s)$ amplitude shape in Fig. (5), considering the perturbed system (29)

from the beneficial properties of the distributed delay, its retarded spectrum of zeros in particular. In future research, the attention will be paid to robustness analysis, spectrum distribution of the perturbed system at the high frequency range and systematic $\mathcal{H}_{\infty}$ optimization of the arising time delay system.

\section{REFERENCES}

[1] T. Inoue, M. Nakano, T. Kubo, S. Matsumoto, and H. Baba, "High accuracy control of a proton synchrotron magnet power supply," IFAC Proceedings Volumes, vol. 14, no. 2, pp. 3137-3142, 1981.

[2] B. A. Francis and W. M. Wonham, "The internal model principle of control theory," Automatica, vol. 12, no. 5, pp. 457-465, 1976.

[3] S. Hara, Y. Yamamoto, T. Omata, and M. Nakano, "Repetitive control system: A new type servo system for periodic exogenous signals," IEEE Transactions on automatic control, vol. 33, no. 7, pp. 659-668, 1988.

[4] J. K. Hale, S. M. V. Lunel, L. S. Verduyn, and S. M. V. Lunel, Introduction to functional differential equations. Springer Science \& Business Media, 1993, vol. 99.

[5] W. Michiels and S.-I. Niculescu, Stability, control, and computation for time-delay systems: An eigenvalue-based approach, 2 nd ed., ser. Advances in Design and Control. Society for Industrial and Applied Mathematics (SIAM), Philadelphia, PA, 2014, vol. 27.

[6] T. Inoue, "Practical repetitive control system design," in 29th IEEE Conference on Decision and Control. IEEE, 1990, pp. 1673-1678.

[7] W. S. Chang, I. H. Suh, and T. W. Kim, "Analysis and design of two types of digital repetitive control systems," Automatica, vol. 31, no. 5, pp. 741-746, 1995.

[8] L. Güvenç, "Stability and performance robustness analysis of repetitive control systems using structured singular values," Journal of Dynamic Systems, Measurement, and Control, 1996.

[9] J.-H. Moon, M.-N. Lee, and M. J. Chung, "Repetitive control for the track-following servo system of an optical disk drive," IEEE transactions on control systems technology, vol. 6, no. 5, pp. 663670, 1998.

[10] T.-Y. Doh and M. J. Chung, "Repetitive control design for linear systems with time-varying uncertainties," IEE Proceedings-Control Theory and Applications, vol. 150, no. 4, pp. 427-432, 2003.

[11] K. Omura, H. Ujikawa, O. Kaneko, Y. Okano, S. Yamamoto, H. Imanari, and T. Horikawa, "Attenuation of roll eccentric disturbance by modified repetitive controllers for steel strip process with transport time delay," IFAC-PapersOnLine, vol. 48, no. 17, pp. 131-136, 2015.
[12] T.-C. Tsao, Y.-X. Qian and, and M. Nemani, "Repetitive control fo asymptotic tracking of periodic signals with an unknown period," $J$. Dyn. Sys., Meas., Control, vol. 122, no. 2, pp. 364-369, 2000.

[13] G. Hillerstrom, "Adaptive suppression of vibrations-a repetitive control approach," IEEE Transactions on Control Systems Technology, vol. 4, no. 1, pp. 72-78, 1996

[14] J.-W. Lin, P. F. Shen, and H.-P. Wen, "Repetitive control mechanism of disturbance cancellation using a hybrid regression and genetic algorithm," Mechanical Systems and Signal Processing, vol. 62, pp. $356-365,2015$.

[15] M. Steinbuch, "Repetitive control for systems with uncertain periodtime," Automatica, vol. 38, no. 12, pp. 2103-2109, 2002.

[16] M. Steinbuch, S. Weiland, and T. Singh, "Design of noise and periodtime robust high-order repetitive control, with application to optical storage," Automatica, vol. 43, no. 12, pp. 2086-2095, 2007.

[17] G. Pipeleers, B. Demeulenaere, J. De Schutter, and J. Swevers, "Robust high-order repetitive control: optimal performance trade-offs," Automatica, vol. 44, no. 10, pp. 2628-2634, 2008.

[18] M. Goubej, T. Vyhlídal, and M. Schlegel, "Frequency weighted h2 optimization of multi-mode input shaper," Automatica, vol. 121, p. $109202,2020$.

[19] T. Vyhlídal, M. Hromčík, V. Kučera, and M. Anderle, "On feedback architectures with zero-vibration signal shapers," IEEE Transactions on Automatic control, vol. 61, no. 8, pp. 2049-2064, 2015.

[20] T. Vyhlídal and M. Hromčík, "Parameterization of input shapers with delays of various distribution," Automatica, vol. 59, pp. 256-263, 2015 .

[21] T. Vyhlídal, V. Kučera, and M. Hromčík, "Signal shaper with a distributed delay: Spectral analysis and design," Automatica, vol. 49, no. 11 , pp. 3484-3489, 2013.

[22] T. Vyhlídal, D. Pilbauer, B. Alikoç, and W. Michiels, "Analysis and design aspects of delayed resonator absorber with position, velocity or acceleration feedback," Journal of Sound and Vibration, vol. 459, p. 114831, 2019.

[23] D. Pilbauer, T. Vyhlídal, and W. Michiels, "Optimized design of robust resonator with distributed time-delay," Journal of Sound and Vibration, vol. 443, pp. 576-590, 2019.

[24] D. Pilbauer, T. Vyhlídal, and N. Olgac, "Delayed resonator with distributed delay in acceleration feedback-design and experimental verification," IEEE/ASME Transactions on Mechatronics, vol. 21, no. 4, pp. 2120-2131, 2016.

[25] C. E. Garcia and M. Morari, "Internal model control. a unifying review and some new results," Industrial \& Engineering Chemistry Process Design and Development, vol. 21, no. 2, pp. 308-323, 1982.

[26] Y.-S. Lu, "Internal model control of lightly damped systems subject to periodic exogenous signals," IEEE Transactions on Control Systems Technology, vol. 18, no. 3, pp. 699-704, 2009.

[27] T. Vyhlídal and P. Zítek, "Control system design based on a universal first order model with time delays," Acta Polytechnica, vol. 41, no. $4-5,2001$

[28] P. Zítek, J. Bušek, and T. Vyhlídal, "Anti-windup conditioning for actuator saturation in internal model control with delays," in LowComplexity Controllers for Time-Delay Systems. Springer, 2014, pp. $31-45$.

[29] S. Skogestad and I. Postlethwaite, Multivariable feedback control: analysis and design. Citeseer, 2007, vol. 2.

[30] W. Michiels and S. Gumussoy, "Computation of h-infinity norms for time-delay systems," TW Reports, 2009.

[31] S. Gumussoy and W. Michiels, "Fixed-order h-infinity control for interconnected systems using delay differential algebraic equations," SIAM Journal on Control and Optimization, vol. 49, no. 5, pp. 22122238, 2011.

[32] M. Overton, "Hanso: a hybrid algorithm for nonsmooth optimization," Available from cs. nyu. edu/overton/software/hanso, 2009.

[33] F. E. Curtis, T. Mitchell, and M. L. Overton, "A bfgs-sqp method for nonsmooth, nonconvex, constrained optimization and its evaluation using relative minimization profiles," Optimization Methods and Software, vol. 32, no. 1, pp. 148-181, 2017.

[34] T. Vyhlídal and P. Zítek, "Qpmr-quasi-polynomial root-finder: Algorithm update and examples," in Delay Systems. Springer, 2014, pp. 299-312. 